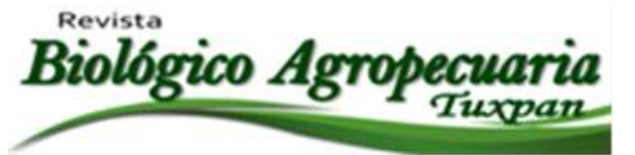

\title{
Afrontamiento por duelo de separación familiar en estudiantes universitarios foráneos
}

Coping with family separation in foreign university students

San Juan Gutiérrez Irene ${ }^{1 凶}$, Higinio Fernández Sánchez², Claudia Beatriz Enríquez Hernández¹, Salomé Pérez Prieto $^{3}$ y Blanca Flor Fernández 1

${ }^{1}$ Facultad de Enfermería de la Universidad Veracruzana, Región Veracruz, Ver, México. ${ }^{2}$ Facultad de Enfermería de la Universidad Veracruzana, Región Poza Rica, Tuxpan, México. ${ }^{3}$ Facultad de Contaduría de la Universidad Veracruzana, Región Veracruz, Ver, México.

${ }^{\square}$ Autor para correspondencia: irenesanjuangtz@hotmail.com

\section{RESUMEN}

En el proceso de adaptación a la vida universitaria los ámbitos social, cultural y académico se ven comprometidos, donde existen cambios afectivos, comportamentales y cognitivos; por lo tanto, factores como el apoyo social, los eventos vitales, la resolución exitosa de problemas prácticos en la nueva cultura y la interacción con ella, son elementos que entran en juego en los estudiantes para el afrontamiento a los problemas. Objetivo: Determinar las estrategias de afrontamiento y la adaptación social de los estudiantes de enfermería que emigraron de su lugar de origen para establecerse en otra región. Métodos: Estudio cuantitativo, no experimental, de tipo descriptivo-transversal con muestreo no probabilístico por conveniencia, con una muestra de 52 participantes. Se aplicó la Escala de Adaptación Social con 21 ítems, con cuatro niveles de respuesta (0 a 3) y Escala Estrategias de CopingModificada con 69 ítems agrupados con 12 estrategias de afrontamiento. Resultados: En cuanto a las estrategias de afrontamiento que perciben los estudiantes, se encontró que el 32.7\% ocupan la solución de problemas con más frecuencia, 50\% ocupa la reacción agresiva en moderada frecuencia. En el proceso de duelo por adaptación social se encontró que el $57.8 \%$ de la población total se encuentran adaptados normalmente y por otra parte el $42.2 \%$ presentan inadaptación moderada. Conclusiones: Las estrategias de afrontamiento son procesos adaptativos que enfrenta el ser humano ante una situación o problemática, que sirven para prevenir, controlar o empeorar el distrés emocional.

Palabras claves: afrontamiento, duelo, adaptación, estudiante de enfermería, separación.

\begin{abstract}
In the process of adapting to college life, the social, cultural and academic fields are compromised where the student will be involved in affective, behavioral and cognitive changes; Therefore, factors such as social support, life events, the successful resolution of practical problems in the new culture and interaction with it, are elements that come into play in students to cope with problems. Aim:
\end{abstract}


Determine coping strategies and social adaptation of nursing students who emigrated from their place of origin to settle in another region. Method: Quantitative study, non-experimental, cross-sectional type with non-probabilistic sampling for convenience, with a 52 sample size. The Social Adjustment Scale instruments were applied with 21 items, with four response levels (0 to 3) and the CopingModified Strategies Scale with 69 items grouped with 12 coping strategies. Results: Regarding the coping strategies that students perceive, it was found that $32.7 \%$ occupy the solution of problems with more frequency, 50\% occupy the aggressive reaction in moderate frequency. In the process of mourning for social adaptation, it was found that $57.8 \%$ of the total population is normally adapted and, on the other hand, $42.2 \%$ have moderate maladjustment. Conclusion: Coping strategies are adaptive processes that face the human being in a situation or problem, which serve to prevent, control or worsen emotional distress.

Keywords: coping, grief, adaptation, nursing student, separation.

\section{INTRODUCCION}

Una de las principales causas de deserción en estudiantes universitarios es el duelo migratorio, lo cual se define como una inadaptación social a los cambios de residencia, (Atxótegui, 2007). Por un lado, la Secretaria de Educación Pública (2016), durante el primer año de universidad los estudiantes foráneos se enfrentan a cambios totalmente nuevos y deben enfrentarlos "solos", produciendo una mayor cantidad de abandono escolar. Además, el Instituto de Estadística y Geografía (2015), reportó que la falta de recursos económicos de los universitarios foráneos para responsabilizarse de su economía, alimentación e higiene, aun así estos los apoyen con el dinero, puede llegar a ser muy difícil para los estudiantes.

En el proceso de adaptación a la vida universitaria y a un nuevo estilo de vida se ven comprometidos los ámbitos social, cultural y académico, en donde el estudiante implicará cambios afectivos, comportamentales y cognitivos (Sam y Berry, 2006, citado por Castro y Lupano, 2013), por lo tanto, factores como el apoyo social, los eventos vitales, la resolución exitosa de problemas prácticos en la nueva cultura y la interacción con ella, son elementos que entran en juego en estudiantes a la hora de hablar del proceso de adaptación (Ward et. al., 2001, citado por Castro y Lupano, 2013).

Por otro lado, aunque el alejamiento de los padres es lo más fuerte (Bowlby, 1998), también lo es alejarse de las personas cercanas y queridas (amigos o pareja) y esto puede llevar a la deserción de los estudios universitarios o a un afrontamiento negativo (Durkheim, citado por García, A. 2015). Aunado al duelo migratorio por separación familiar, los universitarios foráneos pueden llegar a presentar síntomas como es el estrés, tristeza y miedo por lo desconocido.

Ante este panorama, este estudio describe las estrategias de afrontamiento y el duelo migratorio que presentan los estudiantes universitarios foráneos, aunque llegaron a existir otros tipos de problemas en los estudiantes, se identificó que el inicio a la universidad y a lo desconocido provoco un gran impacto en los estudiantes, lo que puede causar la deserción total de los estudios. 


\section{MATERIALES Y MÉTODOS}

El presente trabajo de investigación es cuantitativo, no experimental, de tipo descriptivo-transversal. El cual corresponde a la obtención de los datos en momento específico, la utilidad de este tipo de diseños de estudio consiste en describir variables pocas o nada estudiadas, así mismo se utiliza para establecer la relación de variables en un momento determinado, (García et al., 2014; Hernández et al., 2006; Polit y Hungler, 2000).

La población fue de 120 estudiantes de enfermería de la generación 2017, con una muestra de 52 estudiantes, la cual se obtuvo con un muestreo no probabilístico, a conveniencia, por desconocimiento de la probabilidad de que los elementos fueran seleccionados ya que su elección dependía de las características que se necesitaban para esta investigación; estar cursando el segundo semestre, ser foráneo y estar viviendo en la región de Veracruz.

Los datos se analizaron mediante la estadística descriptiva (frecuencias y porcentajes) utilizando el programa Statistical Package for the Social Sciences (SPSS), versión 21.0 para Windows. Así mismo, los resultados se presentaron a través de tablas.

El instrumento de medición constaba de tres apartados, el primero para obtener los datos sociodemográficos y los que se utilizaron para la evaluación de las variables los cuales fueron: Escala Estrategias de Coping-Modificada (EEC-M) (Londoño et al, 2006).

Esta escala fue elaborada por primera vez por Lázarus y Folkman en 1984 (Ways of Coping Instrument, WCI) (Chorot \& Sandín, 1993). Posteriormente, en 1993, Chorot y Sandín realizaron una versión modificada y mejorada, denominada Escala de Estrategias de CopingRevisado (EECR). Finalmente, Londoño et al. (2006) llevaron a cabo la validación con muestra colombiana llamada Escala de Estrategias de Coping - Modificada (EEC-M). Esta escala tiene como fin evaluar el conjunto de recursos y esfuerzos tanto cognitivos como comportamentales orientados a resolver el problema, a reducir o eliminar la respuesta emocional o a modificar la evaluación inicial de la situación. Consta de 69 ítems, agrupados en 12 factores o estrategias con opciones de respuesta tipo likert, en un rango de frecuencia de 1 a 6 de la siguiente manera: 1, Nunca; 2, Casi Nunca; 3, A veces; 4, Frecuentemente; 5, Casi siempre y 6, Siempre. Los factores o estrategias que componen la escala son: Solución de problemas, Apoyo social, Espera, Religión, Evitación emocional, Apoyo profesional, Reacción agresiva, Evitación cognitiva, Reevaluación positiva, Expresión de la dificultad de afrontamiento, Negación y Autonomía.

Según los resultados psicométricos, la escala cuenta con un Alfa de Cronbach de 0.847, y un $58 \%$ de varianza total acumulada evidenciando que lo evaluado a través de esta prueba está representado por una sola dimensión (estrategia de afrontamiento al estrés), la cual está conformada por 12 factores o estrategias, (Londoño et al., 2006). Por último, la escala se califica sumando los ítems correspondientes a cada estrategia, estas puntuaciones directas se transforman en percentiles para obtener el baremo a partir de la tabla "Media, desviación y percentiles de la Escala de Estrategias de Coping- Modificada (EEC-M) en una muestra colombiana" (Londoño et al., 2006).

Escala Autoaplicada de Adaptación Social (SASS) (Bosc, Dubini, y Polin, 1997). Fue diseñada con el objetivo de evaluar conducta y ajuste social de una forma que permite una gran simplicidad de uso. Se trata de una escala de 21 
ítems, con cuatro niveles de respuesta (de $\mathrm{O}$ a 3 ), que evalúa motivación y conducta social. Los ítems exploran el funcionamiento del individuo en distintas áreas: trabajo, familia, ocio, relaciones sociales y motivación/ intereses. La puntuación total, sumatorio de las puntuaciones de cada ítem, tiene un rango entre O y 60, ya que dos de los ítems son mutuamente excluyentes y cada sujeto sólo responde a uno de ellos en función de si tiene un trabajo u ocupación remunerada o no.

El proceso de validación de la escala SASS consistió en aplicarIa a 4.000 individuos de la población general y, en segundo lugar, a 549 pacientes con depresión mayor. Las puntuaciones de normalidad se sitúan entre $35 \mathrm{y}$ 52 , mientras que las inferiores a 25 suponen una desadaptación social patente. La escala SASS ha mostrado unos índices de fiabilidad y validez adecuados, así como su sensibilidad al cambio durante el tratamiento en pacientes depresivos, la escala tiene cuenta con un Alfa de Cronbach de 0.823 .

La escala se ha utilizado en diferentes investigaciones con población colombiana, como por ejemplo en Martínez et al (2009) y
Ocampo Ramírez, Gaviria Melo, y Muñoz Astudillo (2009); consta de 21 ítems con 4 posibles respuestas cada una, cuyo objetivo es medir el nivel del sujeto en el medio, evaluando seis áreas de funcionamiento del individuo: trabajo, ocio, familia, relaciones sociales, motivación e intereses.

Esta escala ofrece una puntuación total que es la suma de las puntuaciones de todos los ítems sin discriminar las puntuaciones en las áreas mencionadas, por lo tanto los resultados oscilan entre 0 y 60 puntos. Los autores proponen los siguientes puntos de corte son: Hasta 60: Adaptación Normal; 21 - 40: Inadaptación Moderada y 0 - 20: Inadaptación Grave.

\section{RESULTADOS}

Se encuestó a 52 estudiantes que habitan en el estado de Veracruz y provienen de otra región o estado, llamándosele estudiantes universitarios foráneos; comprende en la edad de 18 a 22 años predominando el sexo femenino con el $75 \%$ de la población total y estado civil soltero con el 98.1\% (Tabla 1).

\section{Tabla 1}

Datos sociodemográficos de estudiantes de la Facultad de Enfermería Región Veracruz.

\begin{tabular}{lcc}
\hline Edad & F & $\%$ \\
\hline $18-22$ & 52 & 100.0 \\
Sexo & F & $\%$ \\
Femenino & 39 & 75.0 \\
Masculino & 13 & 25.0 \\
Total & 52 & 100.0 \\
Estado civil & F & $\%$ \\
Soltero/a & 51 & 98.1
\end{tabular}




\begin{tabular}{lcc} 
Unión libre & 1 & 1.9 \\
Total & 52 & 100.0 \\
N. de hijos & F & $\%$ \\
No tienen hijos & 51 & 98.1 \\
1 & 1 & 1.9 \\
\hline Fuente: Directa & & $\mathrm{n}=52$
\end{tabular}

En el nivel de adaptación de los estudiantes en la nueva región y universidad se tiene que el $57.8 \%$ presentan una adaptación favorable o adecuado y solo el $42.2 \%$ obtuvieron inadaptación moderada por lo cual aún están en proceso de duelo (Tabla 2).

\section{Tabla 2}

Nivel de adaptación en estudiantes foráneos de la Facultad de Enfermería Región Veracruz. Adaptación Social

\begin{tabular}{ccc}
\hline & $\mathrm{F}$ & $\%$ \\
\hline Adaptación Social favorable & 30 & 57.8 \\
Inadaptación Moderada & 22 & 42.2 \\
Total & 52 & 100.0 \\
\hline Fuente: Directa & & $\mathrm{n}=52$
\end{tabular}

En lo que comprende a los tipos de estrategias de afrontamiento utilizadas por los estudiantes universitarios foráneos con duelo de separación familiar se encontró que los más utilizados es la solución de problemas $32.7 \%$, en el nivel medio lo ocupa la reacción agresiva con el $50 \%$ y en el nivel bajo o el que menos ocupan es la expresión de la dificultad de afrontamiento lo que corresponde al $38.5 \%$ del total de la población (Tabla 4). 
San Juan et al., 2018

Tabla 3

Estrategias de afrontamiento que utilizan los estudiantes foráneos de la Facultad de Enfermería Región Veracruz.

\section{Estrategias de Afrontamiento}

\begin{tabular}{lcccccc}
\hline & Bajo & $\%$ & Medio & $\%$ & Alto & $\%$ \\
\hline Solución de problemas & 15 & 28.8 & 20 & 38.5 & 17 & 32.7 \\
Espera & 16 & 30.8 & 25 & 48.1 & 11 & 21.2 \\
Religión & 16 & 30.8 & 21 & 40.4 & 15 & 28.8 \\
Búsqueda de apoyo social & 13 & 25 & 24 & 46.2 & 15 & 28.8 \\
Búsqueda de apoyo profesional & 14 & 26.9 & 23 & 44.2 & 15 & 28.8 \\
Reacción agresiva & 14 & 26.9 & 26 & 50 & 12 & 23.1 \\
Evitación cognitiva & 13 & 25 & 23 & 44.2 & 16 & 30.8 \\
Reevaluación positiva & 13 & 25 & 24 & 46.2 & 15 & 28.8 \\
Expresión de la dificultad de & 20 & 38.5 & 20 & 38.5 & 12 & 23.1 \\
afrontamiento & & & & & & \\
Negación & 19 & 36.5 & 18 & 34.6 & 15 & 28.8 \\
Autonomía & 16 & 30.8 & 21 & 40.4 & 15 & 28.8 \\
Evitación emocional & 13 & 25 & 25 & 48.1 & 14 & 26.9 \\
\hline Fuente: Directa & & & & & & $n=52$
\end{tabular}

\section{DISCUSION}

En el presente trabajo de investigación se buscó conocer el tipo de estrategias que perciben los estudiantes universitarios foráneos de la Facultad de Enfermería que presentan duelo migratorio al separarse de sus familiares, donde se encontró que el $32.7 \%$ ocupan la solución de problemas con más frecuencia, 50\% ocupa la reacción agresiva en moderada frecuencia y el $38.5 \%$ ocupa a un bajo nivel de frecuencia la expresión de la dificultad de afrontamiento.
En el proceso de duelo por adaptación social encontramos que el $57.8 \%$ de la población total se encuentran adaptados normalmente y por lo tanto el duelo puede haber sido superado por otra parte el $42.2 \%$ presentan inadaptación moderada por lo que el duelo aún está presente.

Comparando con el trabajo de investigación de Valderrama y Orejuela (2015), ellos encontraron que en la Facultad de Enfermería de la Universidad de Antioquia en Venezuela, el 33\% de la población total (49 estudiantes) ocupan la estrategia de búsqueda de apoyo social con un nivel alto, el $30 \%$ la espera como 
un nivel medio de afrontamiento y el menos usado es el $10 \%$ la reacción agresiva. En la adaptación social se encontró según los autores Valderrama y Orejuela (2015), que el 100\% se encontró con una adaptación social normal.

Por lo anterior se observa que en ambas Universidades algunos estudiantes tienen un afrontamiento negativo, aunque solo se puede llegar a ocupar en ciertas ocasiones no deja de ser una problemática; la espera de que el problema se resuelva solo sin apoyo social y la reacción agresiva podrían ocasionar que el duelo migratorio lleven a una inadaptación social grave.

Según Defaz (2016), en su investigación de las manifestaciones de duelo en los estudiantes migrantes de la Facultad de Psicología de Ecuador, realizó una entrevista donde obtuvo un caso donde el estudiante tuvo dificultad para resolver situaciones de conflicto (expresión de dificultad de afrontamiento), ya que aún estaba el duelo migratorio presente y por lo tanto una inadaptación social, impidiendo la solución de los problemas y creando una dependencia.

A diferencia de los resultados obtenidos con los estudiantes foráneos de la Facultad de enfermería, región Veracruz, la dificultad de la expresión de afrontamiento solo el $23.1 \%$ lo tiene, por lo que puede existir la misma problemática que en el trabajo de investigación de Defaz (2016).

\section{CONCLUSIONES}

En el presente trabajo de investigación se lograron cumplir con los objetivos establecidos; se identificaron los estudiantes universitarios foráneos por medio de la difusión entre compañeros y redes sociales. Se encontró una población total de 52 estudiantes universitarios foráneos en una generación de 120 estudiantes.

Se identificó que los estudiantes están adaptándose al entorno escolar y de la nueva región donde habitan, ya que estuvieron llevando este proceso durante 6 meses antes de ser aplicado los instrumentos de valoración, aunque un porcentaje aún está llevando este proceso de duelo, es un dato significativo que ningún estudiante lleve un proceso de duelo grave.

En los estilos de afrontamiento se encontró que la mitad de la población en estudio aun presentan un afrontamiento negativo como la reacción agresiva, aunque mencionaron que solo se da en pocas ocasiones, esto quiere decir que el proceso de adaptación se va llevando regularmente pero con riesgo de volver a caer en duelo.

Un gran avance que se tiene es que el $32.7 \%$ de la población total llevan el afrontamiento de la solución de problemas siendo esta casi siempre, lo que significa que los estudiantes universitarios están teniendo un aprendizaje adaptativo bueno, ayudándolos a la integración social en esta nueva etapa.

Las estrategias de afrontamiento son procesos adaptativos que enfrenta el ser humano ante una situación o problemática, que sirven para prevenir, controlar o empeorar el distrés emocional (Lipowski, 1970).

Cada año aumentan las demandas en la carrera de Licenciatura en enfermería por lo cual se seguirá recibiendo un gran número de estudiantes universitarios foráneos que dejaran a sus familias, agregándole que es una carrera donde se enfrenta muchos problemas de estrés, el presentar un duelo o una inadaptación social puede provocar aislamiento social y riesgo de suicidios según Durkeim (1965). 
Los estilos de afrontamiento pueden ayudar a los estudiantes a integrarse socialmente, realizando un proceso de adaptación normal y crecen como personas y profesionalmente, pero no siempre las estrategias de afrontamiento pueden ser positivas, al enfrentarse a un problema "solo" desencadenan el duelo migratorio provocando con esto afrontamientos negativos como la reacción agresiva.

Una de las recomendaciones que se considera de primera intención es la inclusión de los estudiantes desde el primer semestre, explicando la importancia del trabajo en equipo, ya que la carrera de enfermería como muchas otras, el éxito se obtiene sabiendo trabajar en armonía con el equipo multidisciplinario. Al integrarlos desde el salón de clases, hará más fácil la adaptación a la nueva región y sociedad donde se encuentra.

Brindar apoyo psicológico como profesionales de la salud ya que presenciar una buena salud mental previene muchas enfermedades causantes por el medio ambiente como: el estrés, la depresión y el aislamiento social.

\section{LITERATURA CITADA}

Atxótegui J., (2001). "Duelo migratorio" Trab. VII Jornadas APAG. Palma de Mallorca.

Bobes. J., González. M., Bascaran. M., Coromina. A. y Adán. A., (1999). "Validación de la versión española de la escala de Adaptación Social en pacientes depresivos". Actas Esp. Psiquiatr, 27(2), 71-80

BOWLBY J., (1985). La separación afectiva. Barcelona: Paidós.

BOWLBY J., (1993). La pérdida afectiva. Tristeza y depresión. Barcelona: Paidós.
Bustos. V., (2013). "El Duelo". Asociación Mexicana de Tanatología, 5-11.

Cassaretto, Chau, Oblitas, Valdez, (2003). "Estrés y afrontamiento en estudiantes de psicología". Pontificia Universidad Católica del Perú, (2).

https://doi.org/10.18800/psico.200302.006

Castro A., Lupano M., (2001). “Adaptación social, diferencias individuales del liderazgo y la cultura organizacional percibida. Facultad de Psicología, Buenos aires, (8).

Castro Solano, A., Lupano Perugini, M., (2013). "Predictores de la adaptación sociocultural de estudiantes universitarios extranjeros en Argentina”. Interdisciplinaria, 30(2), 265-281. https://doi.org/10.16888/interd.2013.30.2.

Chávez Becerra, M., Contreras Gutiérrez, O. Velázquez Ortiz, M., (2013). “Adaptación y pensamiento constructivo en estudiantes universitarios. Psicogente, 16(30), 311323.

Cornejo, Marqueza, Lucero, Mariela Cristina, (2005). "Preocupaciones vitales en estudiantes universitarios relacionado con bienestar psicológico y modalidades de afrontamiento". Fundamentos en Humanidades, (VI), rescatado en: <http://www.redalyc.org/articulo.oa?id $=18412608>$ ISSN 1515-4467.

Cuadrado. D., (2010). "Las cinco etapas del cambio". Trabajo social. (7), 77-97

Defaz. C., (2016). “Análisis de las manifestaciones de la elaboración del duelo". Facultad de psicología. 18-30. ISBN: 978-607-8289-84-4

Donoso. I., (2009). "Consecuencias del desarraigo en los estudiantes universitarios migrantes de la 
Universidad Politécnica Salesiana". Escuela Sistemática. (2), 20-30.

Donoso. M., (2014). "Duelo Migratorio". Asociación Mexicana de Tanatología, A.C., 10-30.

García, A., Adrogué, C., Portilla, A., (2015). "Abandono de los estudios universitarios: dimensión, factores asociados y desafíos para la política pública". Revista Fuentes, 16, Junio, pp. 85-106. https://doi.org/10.12795/revistafuentes.20

González V., (2005). "El duelo migratorio". Departamento de trabajo social, Facultad de ciencias humanas (7), 77-97.

Guevara, Cárdenas, Hernández, (2017). Protocolos de investigación en enfermería, Primera Edición. Manual Moderno, México. ISBN: 978-607-448613-1

Kübler-Ross, (2013). "El significado psicológico de las cinco fases del duelo". Psicoonlogia. (1), 109-130. ISSN: 16967240 .

Lazarus RS, Folkman S. Estrés y procesos cognitivos. Barcelona: Martínez Roca, 1986, (Original de 1984).

Londoño, (2006). "Propiedades psicométricas y validación de la escala de estrategias de coping modificada (EEC-M) en una muestra colombiana". Universitas Psychologica, (2), 327-349, ISSN: 16579267.

Martínez J., (2010). "Estrategias de afrontamiento ante el estrés y rendimiento académico en estudiantes universitarios". Cuadernos de educación y desarrollo, (18).

Martínez, Jiménez, Fernández, (2005). “Estudio sobre la escala de estilos y estrategias de afrontamiento". Universidad Nacional de Educación a Distancia, (4), ISSN: 1138493X.

Phillips, K., (2007). Sor Callista Roy: Modelo de adaptación. En Marriner, A y Raile, M., Modelos y teorías en enfermería.

Soriano. J., (2002). "Reflexiones sobre el concepto de Afrontamiento en Psicología”. Boletín de Psicología. (75), 73-85

Torres Velázquez, Laura Evelia, Rodríguez Soriano, (2006). Norma Yolanda, "Rendimiento académico y contexto familiar en estudiantes universitarios". Enseñanza e Investigación en Psicología, 11 (julio-diciembre), rescatado en: <http://www.redalyc.org/articulo.oa?id $=29211204>$ ISSN 0185-1594.

Tosi, A., (2009). "Migrar para estudiar". Revista Catedra Paralela, (6), ISSN: 1669-8843.

Valderrama. V. y Orejuela. F., (2015). "Adaptación social y estrategia de Afrontamiento en estudiantes de primer semestre de pregrado de la Universidad de Antioquia, sedes de Medellín, provenientes de regiones diferentes a Antioquia". Departamento de Psicología, 27. 
Copyright (c) 2018 Irene San Juan Gutiérrez, Higinio Fernández Sánchez, Claudia Beatriz Enriquez Hernández,

Salom é Pérez Prieto y Blanca Flor Fernández

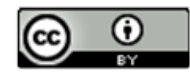

Este tex to está protegido por una licencia licencia Creative Commons 4.0 .

\begin{abstract}
Usted es libre para Compartir — copiar y redistribuir el material en cualquier medio o form ato- y Adaptar el documento —remezclar, transformar y crear a partir del material- para cualquier propósito,, incluso para fines comerciales, siempre que cumpla la condición de:

Atribución: Usted debe dar crédito a la obra original de manera adecuada, proporcionar un enlace a la licencia, e in dicar si se han realizado cambios. Puede hacerlo en cualquier form a razonable, pero no de form a tal que sugiera que tiene el apoyo del licenciante o 10 recibe por el uso que hace de la obra.
\end{abstract}

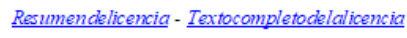

